\title{
Competition Effects in a Young Sitka Spruce (Picea sitchensis, Bong. Carr) Clonal Trial
}

\author{
By S. Brotherstone ${ }^{1), *)}$ I. M. S. White ${ }^{1)}$, R. Sykes ${ }^{2)}$, R. Thompson ${ }^{3)}$, T. Connolly ${ }^{2)}$, S. LeE ${ }^{2)}$ and J. Woolliams ${ }^{4)}$
}

(Received $2^{\text {nd }}$ August 2010)

\begin{abstract}
In the analysis of forestry experiments, there may be a need to adjust for competition between plots before predicting deployment performance in the field but there have been few attempts to investigate this. Our analysis looked at diameter data from a 19-year old Sitka spruce clonal trial growing in Scotland. Using a sequence of nested models, a likelihood ratio test indicated that fitting competition at both the genetic and residual level provided a significantly better fit than models which either ignored competition or fitted it at just the genetic or just the residual level. A strong negative genetic correlation of $-0.93 \pm 0.05$ was found between the direct genetic effects and competition effects. This was not significantly different from -1 , indicating that competition is almost exactly proportional to the direct genetic effect and that a tree will exert a competitive effect which is closely related to its own genetic merit for size. At the residual level, the correlation between direct and competition effect was estimated as $-0.17 \pm 0.03$. We conclude that competition exists at both the genetic and environmental levels and including it in genetic evaluation systems gives a better prediction of future performance. Results also demonstrate that it is possible to obtain useful information about competition effects from a single-tree plot experiment.
\end{abstract}

Key words: genetic variance, genetic prediction, single-plot design, local spatial trends.

\section{Introduction}

In the classical approach to the analysis of agricultural field experiments, the response of each plot is regarded as independent of its neighbours, and neighbour relationships do not feature in the analysis of the experiment. Two developments introduced neighbour relationships into the analysis. First, the need to allow for local trends in fertility not accounted for by the block structure of the experiment (PAPADAKIS, 1937; BARTLETT, 1978). These local trends create positive correlations between yields from neighbouring plots. Second, the need to adjust for interference or competition between

\footnotetext{
1) Institute of Evolutionary Biology, University of Edinburgh King's Buildings, West Mains Road, Edinburgh, EH9 3JT, United Kingdom.

$\left.{ }^{2}\right)$ Forestry Research, Northern Research Station, Roslin, Midlothian, EH25 9SY, United Kingdom.

3) Rothamsted Research, Harpenden, Hertfordshire, AL5 2JQ, United Kingdom.

4) The Roslin Institute, Royal(Dick) School of Veterinary Studies, University of Edinburgh, Roslin Biocentre, Midlothian, EH25 9PS, United Kingdom.

*) Corresponding author: S. BROTHERSTONE. E-Mail: $\underline{\text { s.brotherstone@ed.ac.uk }}$
}

plots (Pearce, 1957; Draper and GutTMan, 1980). In this case correlation between neighbours may be negative.

Models for competition include two effects for each plot: a direct effect, affecting its own yield, as in a conventional analysis, and a competition effect, affecting the yields of its neighbours.

There are two main approaches to modelling the competition effect. One, originally suggested by MEAD (1967), regards the competitive effect of a plot as a linear function of its yield, with the same regression coefficient applying to all plots (KEMPTON, 1982; BESAG and Kempton, 1986; ConNolly et al., 1993). A test of the hypothesis that the regression coefficient is zero determines whether there is evidence for competitive effects. Differences between varieties can be adjusted for the effects of competition, and where appropriate, monoculture yields can be predicted (KEMPTON, 1982). The regression coefficient is essentially the same as that used to detect fertility trends, so any attempt to separate the effects of fertility trends and competition requires a more complex model (DURBAN et al., 2001; STRINGER and Cullis, 2002).

With this approach, analysis of the data should allow for the fact that each plot yield can appear on both left and right sides of the model equation (KЕMPTON, 1982). A simplified version of the analysis ignores this feature, and calculates a competition index for each tree as a (possibly weighted) average of the yields of its neighbours. The competition index is then included as a covariate in the analysis (ANDERSSON et al., 2007).

An alternative approach regards the direct and competition effects as a function of treatment, variety, genotype, or some other feature of the plot (PEARCE, 1957; DRAPER and GUTTMAN, 1980). The competitive effect of a variety (say) on neighbours is estimated separately from its direct effect. Whereas the first approach necessarily deals with competition at the phenotypic level, with the second approach, competition can be regarded as operating at more than one level, e.g. genetic and environmental. We model direct and competition effects as correlated random effects each of which can be split into genetic and non-genetic components following BIJMA et al. (2007a,b) who applied this type of model in the context of animal breeding.

In forestry experiments, once individual trees have reached a critical size, tree-on-tree interaction takes various forms, such as competition for nutrients, light or root space. CANNELL et al. (1984) found evidence of intertree competition effects on height in close-spaced experimental plots of Picea sitchensis and Pinus contorta. 
Competition effects were found to be asymmetrical, i.e. height growth of trees was decreased by the presence of taller neighbours but not by shorter neighbours, suggesting that competition was mainly for light.

CorRell and ANDERSON (1983) used the method of KEMPTON (1982) to adjust for competition in forestry variety trials, and MAGNUSSEN (1989) applied a similar method in a study of jack pine. More recently, de RESENDE et al. (2005) looked at the effect of competition on diameter in a 13-year old Pinus caribaea experiment and an 18-year old Eucalyptus maculata experiment. DeBELl and HARRINGTON (1997) considered the productivity of Populus in monoclonal and polyclonal blocks and concluded that differences among clones in height and diameter were greater in polyclonal than in monoclonal plots. Apart from these examples, there have been few attempts to adjust for competition effects in forestry experiments.

The basic unit of most forestry experiments is a plot of several trees of the same family or treatment. In such experiments we might expect any competition to operate mostly within plots, although competition between trees in different plots is also possible, depending on the layout and geometry of the design. The simplest type of design in which to look for competitive effects is the single-tree plot experiment.

VEEVERS (1978) proposed a design for single tree plots balanced for neighbour effects (the 'beehive' design based on a hexagonal layout). Most forestry trials do not have such balance but it should still be possible to detect competition effects in single-tree plot experiments with conventional designs such as complete or incomplete block designs. At present mature examples of such experiments are rare, but there is a trend in forestry trials towards this kind of design (B. ANDERSSON, personal communication) and the amount of such information should increase in the future.

The objective of this work was to quantify the effect of competition in a single-tree plot experiment and estimate its heritable and non-heritable components. If competition is found to have a significant genetic effect on forest production, including it in breeding value prediction has the potential to increase response to selection even in limited resource conditions.

\section{Materials and Methods}

In 1989, eight one year old Sitka spruce nursery stock plants were randomly selected from 10 available within each of six unrelated full-sib families. In an attempt to yield sufficient cuttings after one growing season, the stock plants were grown in a glasshouse under extended-day conditions using high pressure sodium lights, heat and extra feeding. Fifteen genetically identical cuttings were taken from each of the 48 stock plants and the resulting rooted cuttings planted at Newcastleton forest $60 \mathrm{~km}$ south of Edinburgh near the border with England (55.11N, 2.46W, soil type free draining upland brown earth, accumulated temperature of greater than $5^{\circ} \mathrm{C}$ equal to 1257 day degrees, average annual rainfall $1300 \mathrm{~mm}$, site elevation $180 \mathrm{~m}$ and exposure very shel- tered). Henceforth we refer to the stock plants as 'genotypes' of the experiment.

Trees were planted in single plant plots, with representatives of the 48 genotypes and two controls in each of 15 blocks. Controls were standard for Sitka spruce genetic field trials, i.e., rooted cuttings randomly selected from unimproved direct import material from Queen Charlotte Islands (British Columbia, Canada). The trees were laid out in a rectangular array with 15 rows and 50 columns at $2 \mathrm{~m}$ spacing in both directions. Buffer trees were planted around the boundary so that all trees in the experiment were exposed to similar environmental conditions. For each tree, neighbours were taken as the two adjacent trees in the same row and the two adjacent trees in the same column (four neighbours).

The trait was diameter at 19 years of age although for comparison purposes all analyses were repeated on diameter at the earlier age of 13 years. Of the original 750 trees, 4 were dead by the age of 13 and 28 by the age of 19 years.

Four models were fitted to the data. All models included the effect of genotype as a random effect. Model I included no other terms (corresponding to a standard analysis). Model II included competition at residual but not at genotype level. Model III included competition at genotype level but no residual (environmental) competition. Model IV included competition at both genotype and residual levels. We also fitted an alternative version of Model IV in which the residual covariance structure described above was replaced by a two-dimensional autoregressive spatial structure (AR1 x AR1) in rows and columns, plus a 'nugget' effect. The assumption is that the correlation between two row effects decreases geometrically with the distance separating them, and similarly for columns. The nugget effect represents independent plot error, uncorrelated with row or column effects.

All models were fitted using the program ASReml (GILMOUR et al., 2006). Log-likelihoods were used to compare the goodness of fit of different models. Roughly speaking, a 'good' model has a smaller residual sum of squares and smaller variance components than a 'poor' model. Model IV is essentially that of BIJMA et al. (2007a).

Briefly, associated with each tree is a direct effect (a prediction of performance in the absence of competition from neighbours) and an indirect (competition) effect which acts on each neighbour of the tree. The predicted yield for a tree is then the sum of its own direct effect and the competition effects of its neighbours. Both direct and competition effects have genetic and non-genetic (environmental, residual) components. The residual components (direct $d$ and competition $s$ ) are assumed to be correlated random variables with variances $\sigma_{d}^{2}$ and $\sigma_{s}^{2}$, and covariance $\sigma_{s d}$.

BIJMA et al. (2007a) assume non-overlapping groups of competing individuals, whereas the trees of this experiment can be thought of as arranged in overlapping groups of five interacting trees (one central tree and four neighbours). Because of this, some expressions had to be modified to allow for the slightly different condi- 
tions. For example, effects d and s generate covariances between neighbouring trees, as we now show. Let $\left(d_{1}, s_{1}\right)$ and $\left(d_{2}, s_{2}\right)$ be direct and competition residual effects for two neighbouring trees. The residual covariance between neighbours is $\operatorname{cov}\left(d_{1}+s_{2}, d_{2}+s_{1}\right)=2 \sigma_{s d}$. Вy a similar argument, for any two non-neighbouring trees, the residual covariance is $\sigma_{s}^{2}$ multiplied by the number of shared neighbours $(0,1$, or 2$)$. Thus residual covariances affecting a particular tree do not extend beyond two spacings in any direction. Since competition, as usually understood, involves negative correlations between neighbours, in the model competition is indicated by $\sigma_{s}^{2}>0$ and $\sigma_{s d}<0$. The case $\sigma_{s d}>0$ is more likely to be due to local fertility trends than any form of competition. Thus, evidence for non-genetic competition comes from negative correlations between neighbours and positive correlations between trees with shared neighbours.

In addition, we associate a genetic direct $(D)$ and competition $(S)$ effect with each genotype. These are assumed to be correlated random effects, with variances $\sigma_{D}^{2}$ and $\sigma_{S}^{2}$, and covariance $\sigma_{S D}$. Covariances generated by genetic direct and competition effects are more complicated than those generated by environmental effects. There will be positive genetic correlations between trees with shared neighbours, and (perhaps rarely) negative genetic correlation between neighbouring trees of the same genotype. Whereas the environmental effects act locally, the genetic effects extend over the whole area of the experiment. For example, the direct genetic effects generate positive covariances between trees of the same genotype, and the genetic competition effects generate positive covariance between two trees (perhaps far apart) which have the same genotype of tree as a neighbour.

More details of the model are given in an appendix.

From a breeder's point of view, it is important to note that both components $D$ and $S$ are assumed to be heritable, and contribute to the response to selection.

A special case of model IV occurs when the correlation between genetic direct and competition effects is \pm 1 . The competition effect is then exactly proportional to the direct effect $(S=c D$ for some constant $c$ ). DRAPER and GUTTMAN (1980) considered such models, generally with positive $c$ indicating overlap effects from neighbouring plots. In our case $c$ is negative and indicates competition. The magnitude of $c$ measures the intensity of competition.

\section{Results}

Diameter at 19 years ranged from $8.3 \mathrm{~cm}$ to $28.6 \mathrm{~cm}$, with a mean diameter of $18.9 \mathrm{~cm}$ (standard deviation $4.08 \mathrm{~cm}$ ).

The log-likelihoods of the four models are summarised in Table 1. Starting with Model I (no competition), adding competition effects at either residual (Model II) or genotype level (Model III) significantly improves the fit. Fitting competition at both genotype and residual levels (Model IV) provides a significantly better fit than any other model.

Parameter estimates for all models are also in Table 1. For Model IV, there is a very strong negative correlation between direct and competition effects at the genotype level (-0.93). If this correlation is fixed at -1 , there is no significant reduction in goodness of fit and at the genotype level the model reduces to the form considered by DRAPER and GUTTMAN (1980) with $c=-0.16$. At the residual level, the covariance between neighbours is estimated as $2 \sigma_{s d}=-1.048 \mathrm{~cm}^{2}$ and the corresponding correlation, estimated as $2 \sigma_{s d} /\left(\sigma_{s}^{2}+4 \sigma_{d}^{2}\right)$ is -0.17 s.e. 0.04 . There is no evidence for correlation caused by shared neighbours (i.e. $\sigma_{s}^{2}$ was not significant).

With the alternative version of Model IV, the nugget effect was not significant, and the fit was very similar to that of Model IV, with a statistically significant negative correlation in both row $(-0.23)$ and column $(-0.15)$ directions, in approximate agreement with the correlation generated by competition at the residual level. The estimated variance of the residual competition effect $\left(\sigma_{s}^{2}\right)$ was negative. This suggests that competition at the

Table 1. - Variance components for each of the models, plus the log likelihood of models II - IV relative to model I. Model I has no competition terms, model II includes competition at the residual level only, model III includes competition at the genotype level only and model IV includes competition at both genotype and residual levels. $\sigma_{D}^{2}\left(\sigma_{d}^{2}\right)$ is the direct genetic (environmental) variance, $\sigma_{S}^{2}\left(\sigma_{s}^{2}\right)$ is the competition genetic (environmental) variance and $\sigma_{D S}\left(\sigma_{d s}\right)$ is the covariance between direct genetic (environmental) effects and competition genetic (environmental) effects.

\begin{tabular}{|l|l|l|r|l|}
\hline \multirow{2}{*}{ Component } & \multicolumn{5}{|c|}{ Model } \\
\cline { 2 - 5 } & I & II & III & IV \\
\hline$\sigma_{\mathrm{D}}{ }^{2}$ & $9.03(2.03)$ & $8.23(1.86)$ & $10.30(2.37)$ & $11.27(2.56)$ \\
\hline$\sigma_{\mathrm{S}}{ }^{2}$ & - & - & $0.26(0.09)$ & $0.36(0.11)$ \\
\hline$\sigma_{\mathrm{DS}}$ & - & - & $-1.46(0.40)$ & $-1.88(0.47)$ \\
\hline$\sigma_{\mathrm{d}}{ }^{2}$ & $6.75(0.37)$ & $6.31(0.48)$ & $6.07(0.35)$ & $6.11(0.43)$ \\
\hline$\sigma_{\mathrm{s}}{ }^{2}$ & - & $0.13(0.09)$ & - & $-0.02(0.08)$ \\
\hline$\sigma_{\mathrm{ds}}$ & - & $-0.51(0.12)$ & - & $-0.52(0.11)$ \\
\hline \multicolumn{1}{|c|}{$\operatorname{LogL}$} & 0.00 & 10.2 & 20.3 & 38.2 \\
\hline
\end{tabular}


residual level does not exist. However, we did find residual covariance between neighbours, and this is incompatible with there being no residual competition. It may therefore be more appropriate to model the environmental variation using a two-dimensional autoregressive structure.

\section{Discussion}

We selected diameter for analysis on the basis that it is the trait routinely measured at the stage of growth where competition effects can be expected to operate. Here we concentrated on the diameter measurement at 19 years, the latest assessment available to us. A similar pattern of competition effects was apparent at the earlier diameter assessment of 13 years (data not presented), where the correlation between the direct genetic effect and the competition genetic effect was $-0.94 \pm 0.07$.

It seems likely that similar results will apply to other conifer species, possibly with some variation in the intensity of competition and the age at which it takes effect. For example, the shade-tolerance of Sitka spruce (HARRIS, 1990) might delay the negative effects of competition relative to other species.

Trees may compete at an early stage for water and nutrients, and after canopy closure they compete mainly for light. This competition results in trees growing more slowly than they would in open-growth conditions. If the reduction in growth is spread equally among all trees in a group of neighbours, variation in available resources over an experiment leads to positive correlations in performance between neighbouring trees. Competition, in the sense in which we use the word, is a tendency for reduced growth to be unequally shared among neighbours, and this reduces the size of the positive correlation between neighbours or even makes it negative.

At the genotypic level, the competition effect was found to be almost exactly proportional to the direct effect. A tree with genotype A (which tend to be large) growing next to a tree with genotype $\mathrm{B}$ (which tend to be small) can be expected to require and take more resources, and this effect will inevitably be closely related to the size of the tree. This type of competition is intuitive and unsurprising. A more interesting question is whether there is deviation from proportionality. This would involve (for example) the ability in some cases for a small tree to obtain all and not just some of the resources it needs, at the expense of a neighbouring large tree. We found no evidence for this type of competition.

In this experiment the trees are classified by genotype, and we regard the competition effect as partly genetic. However, it is easy to imagine a similar experiment with trees classified by non-genetic treatment (e.g. ground preparation, fertiliser regime), in which such competition effects might also arise. It seems probable that this form of competition will be associated with any factor directly affecting the size of tree. The competition effect is genetic, but only because it is strongly linked to the genetically determined direct effect.

In our analysis we did not explicitly allow for spatial variation in site properties such as soil fertility or light availability. The effect of such variation is to create positive correlations between neighbouring trees, whereas the effect of (residual level) competition is to create negative correlations. The combined effect of spatial and competition effects is a correlation which could be positive, negative, or zero. However, on the hypothesis of no competition, the correlation is zero or positive, depending on the extent of local spatial variation, and cannot be negative. Therefore the significant negative correlation which we found between neighbours is evidence for (residual level) competition, whether or not spatial trends are present. For the genetic level of competition effects, the aliasing problem is removed by the random positioning of genotypes within the experiment. For these reasons we believe all our results remain valid in the presence of spatial trends. However, since spatial variation is of interest per se, we looked for evidence of such effects by analysing height at 6 years, assumed free from competition. We compared two models, one with and one without a first order autoregressive (AR) process in row and column directions fitted at the residual level. The AR model did not significantly improve the fit of the model (likelihood ratio test, results not shown). We conclude that there is no evidence of local fertility trends at this site. There are signs of global differences (e.g. between blocks), but this is on too large a scale to impact on competition effects.

There is a large body of literature which deals with adjustment for spatial variation. The most popular method is based on the autoregressive process (GILMOUR et al., 2006; DuTKOwski et al., 2002), although other approaches are possible. For example, ANEKONDA et al. (1996) used a Papadakis-type adjustment based on a hexagonal pattern of 6 neighbours.

DRAPER and GUTTMAN (1980) were primarily interested in positive values of the regression coefficient $c$, representing 'overlap' treatment effects. With model IV, we found a correlation at the genotype level close to and not significantly different from -1 . Fixing this correlation at -1 leads to a Draper and Guttman model with $c=-0.16$.

We found evidence of competition effects at both genotype and residual levels. At the residual level, there are two types of correlation: negative correlation between neighbours, and positive correlation between non-neighbouring trees which have shared neighbours. We found evidence for the first but not the second type of correlation. We note that this is incompatible with the residual model used, which does not allow this possibility (the existence of correlation between neighbours implies a non-zero $\sigma_{s d}$, which in turn implies a non-zero $\sigma_{s}^{2}$ ). A separable autoregressive process in row and column directions provided an adequate approximation for correlation between neighbours. The autoregressive model also fits small positive correlations due to shared neighbours.

For a single tree with direct and competition effects $D$ and $S$, the genetic component of its observed phenotype is $G=D+4 \bar{S}$, where $\bar{S}$ is the average genetic competition effect of its neighbours. A standard analysis based on Model I, ignoring competition effects, produces $G$ as a predicted diameter. On the other hand, the genetic contribution of the tree to future production is $T=D+4 S$ 
(the direct effect expressed once, plus its own competition effect expressed on each of its four neighbours). The value of $T$ for a genotype is a prediction of its future performance when grown in monoculture. BIJMA et al. (2007a) call $T$ the 'total breeding value' and propose its use as a measure of genetic merit when competition is taken into account.

The difference, $G-T=4(\bar{S}-S)$, is a measure of the amount by which the standard analysis (unadjusted for competition) misjudges future performance. A genotype of above average competitiveness $(S<\bar{S}$, competition is associated with negative $S$ ) will be overestimated by a standard analysis, and vice-versa. Because of the strong negative correlation between $D$ and $S$, the effect of using $T$ rather than $G$ is to shrink predictions towards zero. In a large experiment, $\bar{S}$ is effectively constant, and $4 \sigma_{s}$ measures the standard deviation of the discrepancy among genotypes. Here $4 \sigma_{s}=2.4$ and figure 1 shows a stem-and-leaf plot of estimates of $G-T$ for the 48 genotypes based on predictions obtained from Models I and IV.

The decimal point is at the $\mid$

$$
\begin{aligned}
-4 & \mid 755 \\
-3 & \mid 995220 \\
-2 & \mid 851 \\
-1 & \mid 985433210 \\
-0 & \mid 8554444433211 \\
0 & \mid 122334455568 \\
1 & \mid 11
\end{aligned}
$$

Figure 1. - Differences between model I and model IV in predicted future performance for each of 48 genotypes.
The distribution is very skew, with many small positive values (representing overestimation by model I) and a long tail of large negative values (Model I understimates). Even though there are some substantial differences, the values are highly correlated with the estimates of $T$. As a result, ranking of genotypes was almost unchanged but, most importantly, absolute values changed. Ranking of genotypes on the basis of $T$ is similar to that based on a standard analysis, but the values of $T$ provide a more realistic prediction of future performance.

However, there is a beneficial aspect to competition. The competition effect, being so strongly correlated to the direct genetic merit, magnifies the genetic differences without upsetting the rankings, and hence improves accuracy of selection. Ranking the best trees will therefore be more accurate, despite the bias, especially in traits of low heritability.

We considered the possibility that there might be genetic variation in sensitivity to the competition effect. To investigate this we regressed diameter on total competition effect separately for each genotype. Figure 2 shows the regression coefficients plotted against the estimated direct effect.

There is a suggestion that the more successful (larger) genotypes are affected more by competition than smaller genotypes. We did not attempt any formal analysis or pursue this further. One possibility, following KEMPTON (1982), would be to fit a multiplicative model where the regression coefficient for tree $\mathrm{Y}$ on tree $\mathrm{X}$ is the product of two terms, one of which measures the size of competition effect and is determined by the genotype of tree X, and the other measures sensitivity to competition and is determined by the genotype of tree $\mathrm{Y}$.

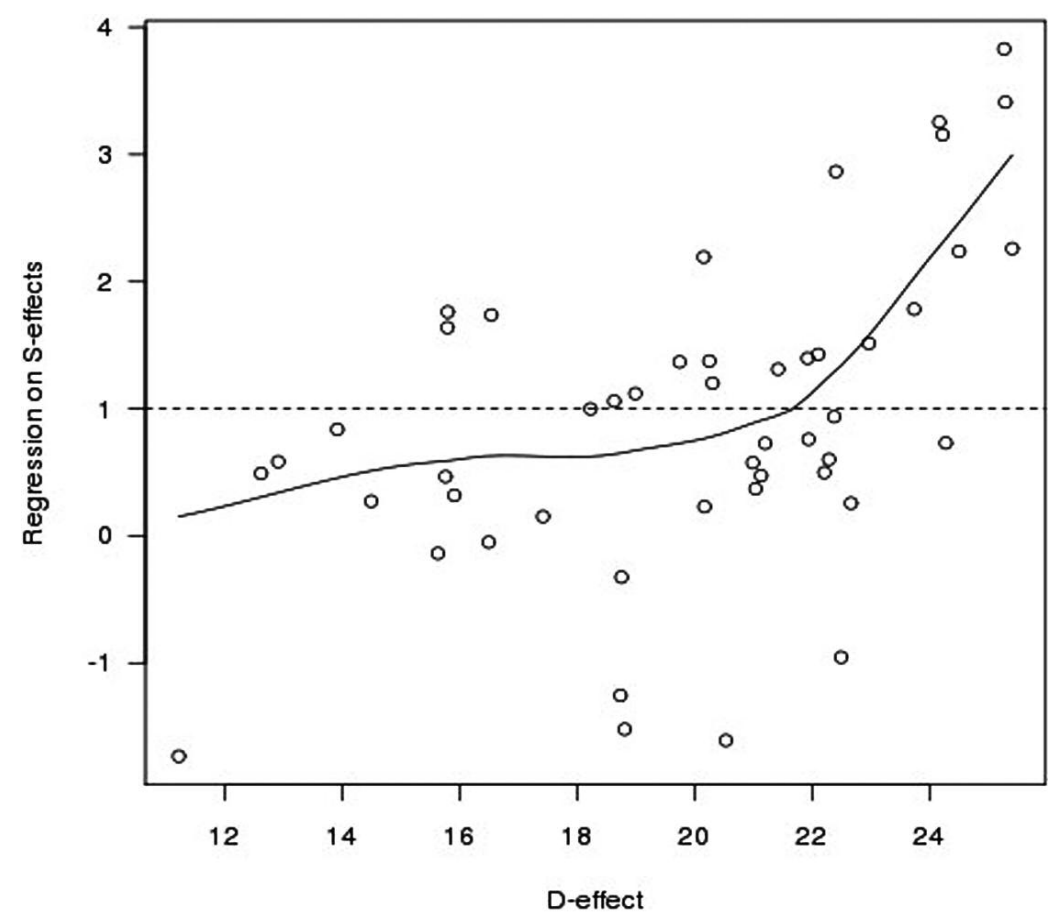

Figure 2. - Regression coefficient for response to competition (Sval) on estimated direct effect (Dval) for diameter at 19 years. The trend curve is a loess smoothing curve. 
We have demonstrated that it is possible to obtain useful information about competition effects from a single-tree plot experiment designed for other purposes. This information would be obtained more efficiently from an experiment specifically designed to look for competition effects, incorporating balanced numbers of neighbours of each genotype, and using hexagonal rather than square spacing (VEEVERs, 1982).

\section{Conclusions}

1. Diameters of trees in a clonal trial show signs of competition at 13 and 19 years.

2. A simple extension to the standard analysis allows competition effects to be measured.

3. The extended analysis gives better predictions of future performance, although the ranking of genotypes may not change substantially.

4. There was a strong correlation between direct and competition effects. Genotypes with large direct effects had large competition effects.

5. Fitting competition effects removes a bias in the breeding value estimation, regressing the estimates of extreme values towards the mean.

6. We suggest that Model IV could be usefully applied in the prediction of genetic merit of conifer trees.

7. The autoregressive process adequately handled competition effects at the residual level.

\section{Acknowledgements}

We thank Bengt Andersson, Tim Mullin and CATHERINE BASTIEN for their many helpful comments and suggestions. Thanks also to the staff of the Forestry Commission who measured the diameter of the trees at 19 years of age specifically for this work. We also thank two anonymous referees for their many helpful and constructive comments. This work has been partially supported by the European Commission within the Seventh Framework Programme for Research (Project Noveltree, FP7-211868).

\section{References}

Andersson, B., B. Elfving, T. Persson, T. Ericsson and J. KROON (2007): Characteristics and development of improved Pinus sylvestris in northern Sweden. Can. J. For. Res. 37: 84-92.

ANEKONDA, T. S. and W. J. LiBBY (1996): Effectiveness of nearest-neighbor data adjustment in a clonal test of Redwood. Silvae Genet. 45: 46-51.

BARTLETT, M. S. (1978): Nearest neighbour models in the analysis of field experiments. J. R. Statist. Soc. B 40: $147-174$

BESAG, J. and R. KEMPTON (1986): Statistical analysis of field experiments using neighbouring plots. Biometrics 42: 231-251.

BiJMA, P., W. M. Muir and J. A. M. VAN AREndonk (2007a): Multilevel selection 1: quantitative genetics of inheritance and response to selection. Genetics 175: 277-288.

Bijma, P., W. M. Muir, E. D. Ellen, J. B. Wolf and J. A. M. VAN ARENDONK (2007b): Multilevel selection 2: Estimating the genetic parameters determining inheritance and response to selection. Genetics 175: 289-299.
Cannell, M. G. R., P. Rothery and E. D. Ford (1984): Competition within stands of Picea sitchensis and Pinus contorta. Annals of Botany 53: 349-362.

Connolly, T., I. D. Currie, J. E. BradshaW and J.W. MCNicol (1993): Inter-plot competition in yield trials of potatoes (Solantum tuberosum L.) with single-drill plots. Ann. Appl. Biol. 123: 367-377.

CoRRELL, R. L. and R. B. ANDERson (1983): Removal of intervarietal competition effects in forestry variety trials. Silvae Genetica 32(5-6): 162-165.

DeBell, D. S. and C. A. HaRrington (1997): Productivity of Populus in monoclonal and polyclonal blocks at three spacings. Can. J. For. Res. 27: 978-985.

DE Resende, M. D. V., J. Stringer, B. Cullis and R. THOMPSON (2005): Joint modelling of competition and spatial variability in forest field trials. Rev. Mat. Estat. Sao Paulo, v. 23, n.2, 7-22.

DRAPER, N. R. and I. GUTTMAN (1980): Incorporating overlap effects from neighbouring units into response surface models. Appl. Statist. 29: 128-134.

Durban, M., Currie, I. D. and Kempton, R. A. (2001): Adjusting for fertility and competition in variety trials. J. Ag. Sci. 136: 129-140.

Dutkowski, G.W., J. Costa e Silva, A. R. Gilmour and G. A. Lopez (2002): Spatial analysis methods for forest genetic trials. Can. J. For. Res. 32: 2201-2214.

Gilmour, A. R., B. J. Gogel, B. R. Cullis and R. ThompSON (2006): ASReml User Guide Release 2.0 VSN International Ltd., Hemel Hempstead HP1 1ES, UK.

HARRIS, A. S. (1990): Picea sitchensis. In: BuRns, R. M. and Honkala, B. H., Silvics of North America Vol. 1, Conifers. Washington DC: U.S.D.A. Forest Service Agriculture.

KEMPton, R. A. (1982): Adjustment for competition between varieties in plant breeding trials. J. Agric. Sci. 98: 599-611.

LYNCH, M. and B. WALSH (1998): Genetics and Analysis of Quantitative Traits. Sinauer Associates, Sunderland.

MAGNUSSEN, S. (1989): Inter-plant interactions and their influence on within and among plot variances. Scand. J. For. Res. 4: 369-377.

MEAD, R. (1967): A mathematical model for the estimation of inter-plant competition. Biometrics 23: 189-205.

PAPADAKIS, J. S. (1937): Méthode statistique pour des expériences sur champ. Bull. Inst. Amél. Plantes à Salonique No 23.

PeARCE, S. C. (1957): Experimenting with Organisms as Blocks. Biometrika 44: 141-149.

StRINGeR, J. K. and B. R. Cullis (2002): Application of spatial analysis techniques to adjust for fertility trends and identify interplot competition in early stage sugar cane trials. Austr. J. of Agric. Res. 53: 911-918.

VEEVERS, A. (1982): Balanced designs for observing intravariety nearest-neighbour interactions. Euphytica 31: $465-468$.

\section{Appendix}

Model IV is

$y=m+(D+\Sigma S)+(d+\Sigma s+e)$

where y is the measured diameter for a tree, $D$ and $d$ are genetic and environmental components of the direct 
effect, $S$ and $s$ are genetic and environmental components of the competition effect, $e$ is the residual, representing environmental variation between clones of the same genotype. Summations are over the four neighbours of the tree. The brackets group genetic and nongenetic terms of the equation. In Model I, where only a genetic and a residual effect are fitted, the genetic effect is equivalent to $(D+\Sigma S)$, where $\Sigma S$ is the sum of neighbour genetic competition effects, whereas performance in monoculture is determined by the value of $D+4 S$, where $S$ is the tree's own genetic competition component.

Equation (1) represents a random effects model. The only non-standard feature is the fact that each measurement is affected by more than one competition effect. To deal with the genetic competition term, for example, we 'overlay' the effects of four factors, each of which represents the genotype of one neighbour (e.g., north, east, south and west of the target tree). There is an analogy with the analysis of a diallel cross experiment, where the value of each cross is the sum of two line effects, one from the male and one from the female parent.
As already mentioned, we allow for covariances between $D$ and $S$, and between $d$ and $s$. Environmental covariances between $d$ and $s$ have been discussed above. The covariance between $D$ and $S$ (the direct and competition effects for the same tree) is $\sigma_{S D}$. This is also the covariance between $S$ and $D$ for two trees of the same genotype. Using standard results on resemblance between relatives, the covariance between $D$ for a tree of one genotype and $S$ for tree of a different genotype is $(1 / 2) \sigma_{S D}$ if the two genotypes belong to the same family (see page 4 for details of the family structure), and is otherwise zero. Similar results are obtained for covariances between values of $D$ or $S$ for two trees by replacing $\sigma_{S D}$ by $\sigma_{D}^{2}$ or $\sigma_{S}^{2}$.

These covariance structures are easily included in the mixed model, either by including additional random effects (family, genotype) in the model, or more directly by calculating a relationship matrix for the 48 genotypes and using the so-called 'animal' model.

A useful reference for the mixed and animal models is LYNCH and WALSH (1998).

\title{
Induction of $2 \mathrm{n}$ pollen by colchicine in Populus $\times$ popularis and its triploids breeding
}

\author{
By X. J. XI ${ }^{1,2), 3), \$)}$ X. B. JIANG ${ }^{1), 2), 3), \$)}$, D. LI ${ }^{1,2), 3)}$, L. Q. GUO' ${ }^{1), 2), 3)}$ \\ J. F. ZHANG ${ }^{1), 2), 3), *)}$ Z. Z. WeI ${ }^{1,2), 3), 4)}$ and B. L. $\mathrm{LI}^{1,2), 3), 5), *)}$
}

(Received $22^{\text {th }}$ November 2010)

\begin{abstract}
Induction of $2 \mathrm{n}$ pollen is a required technique for cultivating polyploid via sexual polyploidy. Orthogonal design or Taguchi Design was applied to select the best treatment process of $2 \mathrm{n}$ pollen induction in Populus $\times$ popularis from different levels of the meiosis stage of

1) National Engineering Laboratory for Tree Breeding, Beijing Forestry University, 100083, Beijing, P. R. China.

$\left.{ }^{2}\right)$ Key Laboratory of Genetics and Breeding in Forest Trees and Ornamental Plants, Ministry of Education, Beijing Forestry University, 100083, Beijing, P. R. China.

3) The Tree and Ornamental Plant Breeding and Biotechnology Laboratory of State Forestry Administration, Beijing Forestry University, 100083, Beijing, P. R. China.

4) Vegetable Research Center, Beijing Institute of Agriculture and Forestry, 100097, Beijing, P. R. China.

5) Department of Forestry and Environmental Resources, North Carolina State University, Raleigh, 27695, North Carolina, USA.

*) Corresponding authors:

Jinfeng Zhang, Beijing Forestry University, Campus Box 118 , Beijing, 100083, P. R. China. Phone: 0086-10-62338415, fax 0086-10-62338013. E-Mail: zjf@bju.edu.cn.

BAILIAN LI, North Carolina State University, 329 Daniels Hall, Campus Box 7112, Raleigh, NC, 27695-7112, USA. Phone 919515-3201, fax 919-515-6835. E-Mail: Bailian_Li@ncsu.edu.

$\$$ ) These authors contributed equally to this work.
\end{abstract}

male flower buds, colchicine concentration, times of injection, and interval between injections. Flow cytometry and chromosome counting were used to identify the triploids from the offspring of $P$. $\times$ euramericana. (Dode) Guinier pollinated with induced pollen of $P . \times$ popularis. The results showed that high $2 \mathrm{n}$ pollen rate can be achieved by selecting the flower buds during diakinesis stage in meiosis, and then injecting $0.6 \%$ colchicine 4 times with 2 hours interval. The $2 \mathrm{n}$ pollen rate reached $62.10 \%$ by this process, and two triploids were obtained, which indicates that it is possible for cultivating triploids via $2 \mathrm{n}$ pollen induction by colchicine treatment in poplar. Results and protocol related to $2 \mathrm{n}$ pollen induction, polyploid identification and effect of $2 \mathrm{n}$ pollen in this study might be applicable in polyploidy breeding in section Aigeiros and Tacamahaca of poplar.

Key words: 2n pollen, poplar, polyploid breeding, colchicine, Orthogonal design.

\section{Introduction}

Polyploidy (having three or more complete sets of chromosomes) is considered to be a major pathway for plant evolution and can result in reproductive isolation and abrupt speciation (RAMSY and SCHEMSKE, 1998; Soltis et al., 2004; WENDEL, 2000). The effects of polyploidy on plant traits are also important to tree or plant 